\title{
Nanomaterial-assisted laser desorption ionization for mass spectrometry-based biomedical analysis
}

Nanomaterials have been widely used to assist laser desorption ionization of biomolecules for mass spectrometry analysis. Compared with classical matrix-assisted laser desorption ionization, strategies based on nanomaterial-assisted ionization generate a clean background, which is of great benefit for the qualitative and quantitative analysis of small biomolecules, such as therapeutic and diagnostic molecules. As label-free platforms, they have successfully been used for high-throughput enzyme activity/inhibition monitoring and also for tissue imaging to map in situ the distribution of peptides, metabolites and drugs. In addition to widely used porous silicon nanomaterials, gold nanoparticles can be easily chemically modified by thiol-containing compounds, opening novel interesting perspectives. Such functionalized nanoparticles have been used both as probes to extract target molecules and as matrices to assist laser desorption ionization for developing new enzyme immunoassays or for studying DNA hybridization. More recently, semiconductor nanomaterials or quantum dots acting as photosensitive centers to induce insource redox reactions for proteomics and to investigate biomolecule oxidation for metabolomics have been shown to offer new analytical strategies.

KEYWORDS: gold nanoparticle ionization laser desorption mass spectrometry mass spectrometry imaging photo-oxidation/reduction porous-silicon titania

\section{Nanomaterial-assisted laser desorption ionization}

Since the introduction of two soft ionization methods, namely matrix-assisted laser desorption/ionization (MALDI) [1] and electrospray ionization [2], mass spectrometry (MS) has developed as one of the most important characterization tools for biomolecules, including proteins, such as nucleic acids and metabolites, among others. MALDI is a subgroup of laser desorption ionization (LDI), a technology that can transfer solid-state molecules to gas-phase ions by illuminating the analytes with a laser. For a typical MALDI process, matrices, usually organic molecules that can absorb UV light and form cocrystal with the analytes, are mixed with the analytes in solution, and the mixture is deposited on a stainless steel target plate. Under UV laser irradiation, the matrices can accumulate energy and can be desorbed together with the embedded analytes in a vacuum system. Ionization of analytes results from charge transfer reactions taking place either directly in the solid phase or in the plume formed by the laser pulse [3]. Charged ions are then extracted by an electric field and separated in the mass analyzer. Owing to the presence of matrices, the analytes cannot be directly irradiated and destroyed by the laser; therefore, intact molecule ions can be produced.
Matrix-assisted laser desorption/ionization mass spectrometry is an excellent method for the analysis of large biomolecules. However, mass ranges under $700 \mathrm{Da}$ are usually seriously impeded by the presence of matrix cluster ions, therefore limiting the application of MALDI in the characterization of small molecules, such as therapeutic molecules. To solve this problem, inorganic materials have been developed to assist LDI of low-molecular-weight analytes. These materials should be able to absorb light energy in accordance with the laser wavelength and should not generate ions that can be detected by the MS. The first example of inorganic material-assisted LDI is indeed the initial work reported by Tanaka et al. in 1987, where glycerol and cobalt nanopowder were employed as absorbers of the laser energy [4]. In this article, we name this inorganic material-assisted LDI as matrix-free LDI. Specifically, when inorganic nanostructure material, such as nanoparticles, nanoporous materials or nanowires, is used to assist the LDI, the ionization method is named as nanomaterial-assisted LDI (NALDI).

In 1999, an important matrix-free LDI method was introduced by the Siuzdak group performed by depositing analytes directly on a porous silicon substrate. The technique was named desorption/ionization on porous silicon (DIOS), and is illustrated in Figure 1 [5]. Compared with

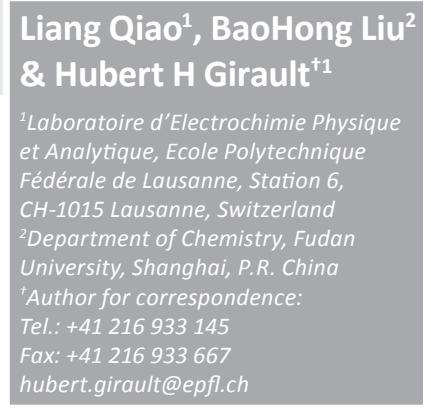

future medictio $_{\text {fsg }}$ fs 


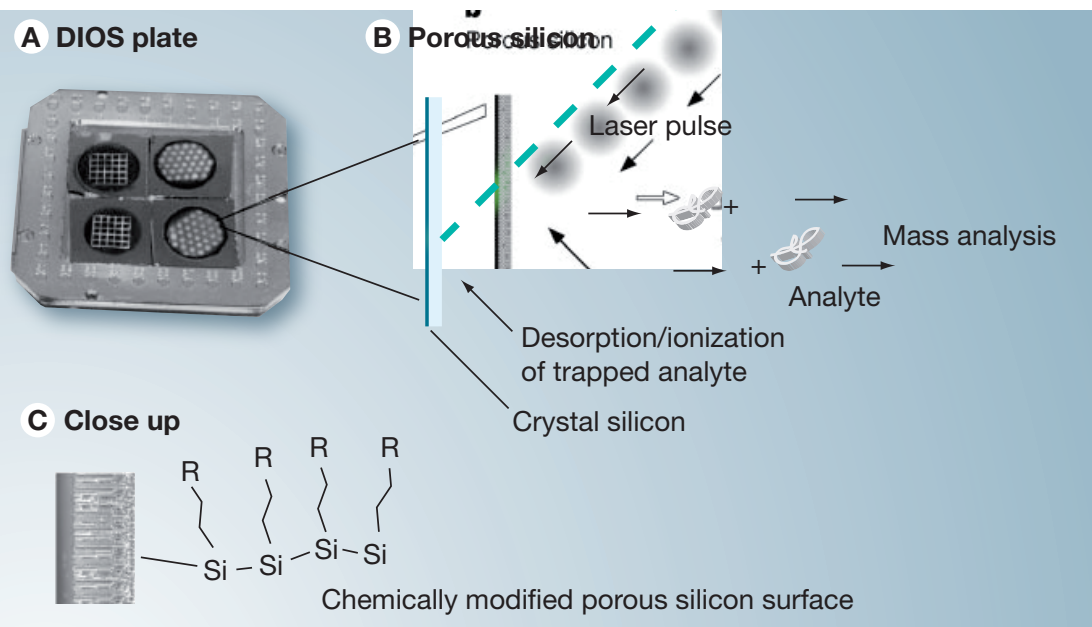

Figure 1. Experimental configuration for desorption/ionization on porous silicon mass spectrometry experiments. (A) Four porous silicon plates are placed on a matrix-assisted laser desorption ionization plate. (B) The silicon-based laser desorption/ionization process, in which the sample is placed on the porous silicon plate and allowed to dry, followed by laser-induced desorption/ionization mass spectrometry. (C) Cross-section of porous silicon, and the surface functionalities after hydrosilylation.

DIOS: Desorption/ionization on porous silicon; R: Penyl or alkyl chains; Si: Silicon. Reproduced with permission from [5].
MALDI, DIOS shows a very clean background in the low mass range. Examples of DIOS-MS on peptides, caffeine, reserpine and $N$-octyl $\beta$-Dglucopyranoside were shown [5]. Sensitivity of 700 attomol of peptide was achieved [5]. It was reported that both n-type mesoporous (2-50 nm pore size) silicon and p-type micro- $(\sim 2 \mathrm{~nm})$ or meso-porous silicon were effective in generating signals, but those surfaces with smaller pore sizes typically gave a more intense ion signal [5]. Based on this DIOS method, various siliconrelated NALDI strategies have been reported, such as atmospheric pressure DIOS [6-9], silicon nanoparticle-assisted laser desorption/ionization [10], infrared laser DIOS [11], desorption/ ionization on silicon nanowires [12], desorption/ ionization on mesoporous silicate [13,14] and desorption/ionization on porous silicon dioxide [15]. The applications of DIOS-MS include qualitative and quantitative analysis of small molecules, especially drugs, metabolites and in situ ionization of small molecules for tissue MS imaging.

In addition to porous semiconducting materials, gold nanomaterials have been employed to assist LDI [16-18]. Compared with silicon, gold nanoparticles (AuNPs) can be chemically modified by self-assembled monolayers [19], and therefore functionalized for various purposes. In one example, AuNPs were capped with 4-aminothiophenol and the modified AuNPs were employed for LDI-MS [20]. It was demonstrated that the capped nanoparticles could increase ion yields, decrease ion fragmentation and increase the useful analyte mass range (up to $\sim 12,000 \mathrm{Da}$ ) when compared with bare AuNPs [20]. AuNPs were also modified with $\alpha$-cyano-4-hydroxycinnanic acid, a typical organic matrix, for effective NALDI measurements [21]. It was found that the $\alpha$-cyano-4-hydroxycinnanic acid-terminated AuNPs showed marked improvement on peptide ionization compared with citrate-capped or cysteamine-capped AuNPs. In addition, the $\alpha$-cyano-4-hydroxycinnanic acid coating on AuNPs effectively suppressed formation of $\mathrm{Au}$ cluster ions and analyte fragment ions, leading to a cleaner mass spectra. Another special property of AuNPs is their ability to absorb visible light owing to localized surface plasmon resonance (SPR) and visible-LDI with the assistance of AuNPs was developed [22-24]. Normally, a $532-\mathrm{nm}$ visible laser, corresponding to the SPR of approximately $15 \mathrm{~nm}$ diameter gold nanospheres, is used instead of the typical UV laser of 337 and $355 \mathrm{~nm}$. The visible laser NALDI may show advantages in the analysis of compounds sensitive to UV light.

Another interesting material for NALDI is nanotitania [25-28]. $\mathrm{TiO}_{2}$ semiconducting nanoparticles are widely used in the field of photocatalysis [29] and for the design of dye-sensitized solar cells. Under UV light irradiation, electrons in the valence band of $\mathrm{TiO}_{2}$ can be excited into the conduction band, and both holes and electron can be trapped at the nanoparticle surface to form oxidative holes and reductive electrons able to react with surrounding molecules [30-32]. Using nano- $\mathrm{TiO}_{2}$ to assist $\mathrm{LDI}$, in-source redox reactions induced by these redox centers can be observed during the ionization processes [30-32].

Other inorganic materials are also used to assist LDI, such as graphite [33], carbon nanomaterials [34-36], nanodiamonds [37], porous alumina [38], nano- $\mathrm{ZnO}[39,40], \mathrm{EuF}_{3}$ hollow hexagonal nanodisks [41], silver nanoparticles [42], $\mathrm{HgTe}$ nanostructures [43], platinum nanoflowers [44], manganese oxide nanoparticles [45], $\mathrm{Fe}_{3} \mathrm{O}_{4}$ particles [46], fullerene-derivatized silica [47] and germanium nanodots [48], among others. Recently, we have shown that quantum dots (QDs) of cadmium selenide and cadmium selenide covered with cadmium sulfide can also be used to assist LDI [49]. Several of the most important publications regarding inorganic material-assisted LDI are listed in TaвLE 1 with descriptions.

Generally, NALDI shows a cleaner background in the low mass range and more homogeneous surface geometry suitable for quantitative 
analysis than the typical MALDI. Furthermore, these nanoparticles are easily modified and functionalized to selectively capture and ionize specific analyte samples. However, various nanomaterials can influence the analytical results in terms of sensitivity, mass range and reproducibility. Chiang et al. have compared the ionization performance of AuNPs, $\mathrm{TiO}_{2} \mathrm{NPs}$, SeNPs, CdTe QDs, $\mathrm{Fe}_{3} \mathrm{O}_{4} \mathrm{NPs}$ and platinum nanospongeassisted LDI. They reported that AuNPs were useful for small analyte ionization while platinum nanosponges and $\mathrm{Fe}_{3} \mathrm{O}_{4} \mathrm{NPs}$ were efficient for protein ionization (up to $25 \mathrm{kDa}$ ) [50]. Shenar et al. compared peptide LDI efficiency on supports of pencil lead, porous silica gel, DIOS-chip and NALDI ${ }^{\mathrm{TM}}$ target, and showed that chromatography porous silica gel was more attractive than existing reference supports for sensitive peptide detection [51]. Tang et al. investigated the relationship between ion desorption efficiency and internal energy transfer in carbonbased surface-assisted LDI, including carbon nanotubes, buckminsterfullerene $\left(\mathrm{C}_{60}\right)$, nanoporous graphitic carbon, nonporous graphite particles, highly oriented pyrolytic graphite and nanodiamonds. It was found that the desorption efficiency generally exhibits an opposite trend to the extent of internal energy transfer [52]. In this article, we mainly focus on the recent application in biomedical study of matrix-free LDI methods, especially some important progresses since 2006, including biomolecule qualitative and quantitative analysis, LDI-MS tissue imaging, nanoparticle-based enzyme immunoassays/DNA hybridization and LDI in source photo-reaction with biomolecules. Complementary information on nanomaterial-assisted LDI for biomolecule analysis can be found in published reviews [53,54].

\section{NALDI in qualitative, quantitative \& imaging analysis of small molecules}

The most important application of NALDI is the characterization of small molecules, such as lipids, fatty acids, metabolites, drug molecules and enzymatic products, for the development of therapeutic and diagnostic strategies [55-61].
Furthermore, with experimental optimization, NALDI was proven to be capable of performing quantitative analysis [62], therefore opening new opportunities for studying enzyme activity and on-tissue medical imaging.

Siuzdak et al. demonstrated DIOS-MS as a quantitative analytical tool in the analysis of small peptides [63]. Electrospray deposition was used to improve sample homogeneity across the porous silicon surface, and therefore to improve the quantitative analysis [63]. A linear relationship between the mass spectra peak intensity and concentration ratios (analyte/internal-standard) in the logarithmic scale was observed. DIOS-MS was further employed for the quantification of codeine, where acceptable accuracy and precision were obtained by using DIOS-MS quantification compared with standard liquid chromatographyMS methods [64]. Similarly, other nanomaterials were also employed to perform quantitative analysis. Oxidized carbon nanotubes were tested as matrices for quantitative analysis of small molecules by MS [65]. Compared with nonoxidized carbon nanotubes, oxidized carbon nanotubes can facilitate sample preparation owing to their higher solubility in water. Moreover, the matrix layer of oxidized carbon nanotubes is much more homogeneous and compact than nonoxidized carbon nanotubes. Accordingly, the reproducibility of peak intensities within and between sample spots was greatly enhanced on the surface of oxidized carbon nanotubes. Quantitative analysis of jatrorrhizine and palmatine with a linear range of 1 to $100 \mathrm{ng} / \mathrm{ml}$ was achieved. Other typical examples of NALDI-MS quantitative analysis include the analysis of glucose from blood plasma using titania nanotubes as matrix [66] and the quantification of glucose in human urine by using AuNPs as matrix [67], which can be important for the diagnosis of diabetes.

Besides the direct characterization of metabolites and drug molecules, NALDI-MS was also used for the investigation of enzyme activity and inhibition. MS-based enzyme assay is a label-free method, therefore avoiding the laborintensive tagging processes classically used in

\section{Table 1. Important published matrix-free laser desorption ionization methods.}

\begin{tabular}{|lllr|}
\hline Material & Year & Description & Ref. \\
\hline Cobalt nanopowder & 1987 & The breakthrough for large molecule LDI & {$[4]$} \\
\hline Carbon & 1995 & Graphite-assisted LDI & {$[33]$} \\
\hline Porous silicon & 1999 & Most widely used matrix-free LDI method & {$[5]$} \\
\hline Gold clusters & 2004 & Developed from secondary ion mass spectrometry & {$[16]$} \\
\hline Titania & 2004 & Titania sol-gel-assisted LDI & {$[27]$} \\
\hline LDI: Laser desorption ionization. & & \\
\hline
\end{tabular}


radioactivity- or fluorescence-based techniques. Siuzdak et al. employed DIOS chips (96 or 384 dots) to run high-throughput DIOS-MS to monitor enzyme activity and enzyme inhibition $[68,69]$. Inhibitors from a library were characterized and their activities against selected enzyme targets, including phenylalanine hydroxylase, glycotransferase and acetylcholinesterase, were monitored. For phenylalanine hydroxylase, phenylalanine was selected as substrate, and tyrosine was produced. For glycotransferase, $\alpha-(2,6)$ sialylated trisaccharide was produced from the corresponding lactoside. For acetylcholinesterase, choline was generated from acetylcholine. The inhibition was determined by monitoring the substrate-to-product peaks ratio on the mass spectra. On two different commercially available instruments, a sampling rate of up to 38 inhibitors per min was accomplished, with thousands of inhibitors being monitored.

Reaction equilibrium can be monitored by NALDI-MS as well. Powell et al. used DIOS-MS to investigate the binding affinities between aldopentose isomers and boron [70]. 1,4-anhydroerythritol was firstly used to compete with individual aldopentoses in forming borate complexes to determine the binding preference among the four aldopentoses. Peak intensities of different complexes were used to identify the quantity of each compound. Afterwards, ${ }^{13} \mathrm{C}$-labeled ribose was included in another set of competition experiments to further confirm the first competition results. The purpose of using ${ }^{13} \mathrm{C}$-labeled ribose is to eliminate the differences in ionization efficiency of various complexes induced by the differences in chemical structures. Ribose exhibited higher affinity to boron than other aldopentoses, and the binding preference was demonstrated to be ribose $>$ lyxose $>$ arabinose $>$ xylose. The result indicates that the favored binding between ribose and boron can be an important factor in RNA evolution. There are several reasons for using DIOS to perform such reaction equilibrium investigation. Besides the advantages, such as a clean background in the low mass range and a good reproducibility of signal intensity, DIOS-MS can avoid the use of organic matrices, which might change the $\mathrm{pH}$ condition of the reaction system and disturb the equilibria.

Mass spectrometry imaging has been used as a label-free method to investigate the in situ distribution of peptides, proteins, metabolites and medicines in tissues and cells [71]. The most popular imaging mass spectrometers involve MALDI-MS, LDI-MS, secondary ion MS and desorption electrospray ionization MS [71]. The theoretical and instrumental aspects, together with the applications of MS imaging, have been reviewed in recent publications [71-73]. A typical MS imaging process comprises the acquisition of mass spectra for a regular series of points across a section of tissue and then the plot of the relative intensities of individual $\mathrm{m} / \mathrm{z}$ data across the tissue, thus visualizing the distribution of the individual molecules. NALDI-MS has also been employed to carry out MS imaging, especially for small molecules. Compared with MALDI-MS imaging, NALDI can avoid the decrease of spatial resolution that is induced by the matrix crystal size and the migration of analytes on the tissue surface during the matrix-depositing process. Since nanomaterials can usually generate a very homogeneous and regular surface, accurate quantification results can be obtained. He et al. used DIOS-MS to map small molecules on mouse liver tissues [74]. In addition, phosphatidylcholine and propidium iodide were used as cell membrane and nucleus markers, respectively, to 'visualize' the presence of HEK 293 cells. The tissue was cut into thin slices $(50 \mathrm{~nm})$ and then transferred onto a DIOS substrate for NALDI-MS imaging of the molecules of interest. As an alternative strategy, Setou et al. sprayed nanoparticles on the tissue surface to visualize peptides and lipids at a resolution of $15 \mu \mathrm{m}$ in mammalian tissues [75,76]. Recently, Volny et al. reported an interesting MS imaging protocol using the commercial NALDI target plate, as shown in Figure 2 [55]. NALDI surfaces were used first as substrates for imprinting of tissue sections, and the transferred lipids were then washed and imaged by LDI-MS.

\section{Nanomaterials as both probes to extract biomolecules \& matrices to assist LDI}

Functionalized nanomaterials can be used as probes to enrich molecules of interest, or to immobilize antibodies and DNA. The extracted molecules on the nanomaterials can then be directly ionized under laser irradiation and analyzed by MS. With this strategy, investigations, such as selective characterization of low-abundance molecules, MS-based enzyme immunoassay and MS-based DNA hybridization assay, were performed. As examples, $\mathrm{TiO}_{2}, \mathrm{Al}_{2} \mathrm{O}_{3}$ and $\mathrm{ZrO}_{2}$ were used to extract phosphorylated peptides from protein digests, and the enriched phosphopeptides were then analyzed directly by MALDI or NALDI-MS [66,77-81]. Titania nanoparticles were also used as selective probes and matrices for the determination of catechins in tea using NALDI-MS [82]. 


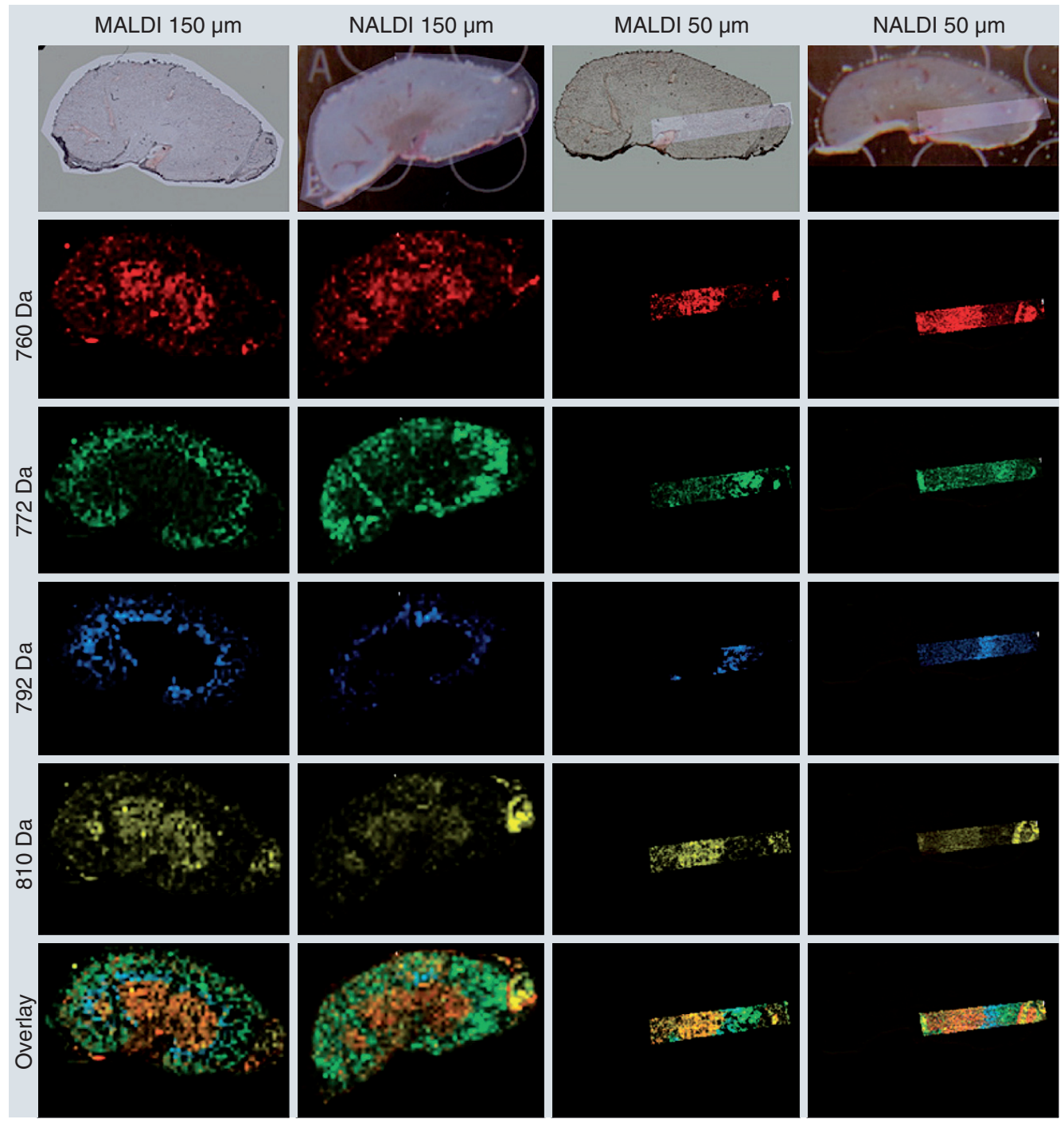

Figure 2. Comparison of standard matrix-assisted laser desorption/ionization imaging with the matrix-free nanomaterial-assisted laser desorption/ionization approach. The overlay images (last row) show the different lipid composition of pelvis (orange) and adrenal gland (yellow) from the rest of the kidney.

MALDI: Matrix-assisted laser desorption ionization; NALDI: Nanomaterial-assisted laser desorption ionization.

Reproduced from [55] with permission from the American Chemical Society.

Among various nanomaterials, gold is indeed one of the most suitable for functionalization as it can easily bind thiol molecules. In a simple application, AuNPs nanoparticles were used to extract aminothiols, including glutathione, cysteine (Cys), and homocysteine [83,84]. The enriched aminothiols were directly ionized on the surface of AuNPs for qualitative and quantitative analysis. With this selective preconcentration approach, the limits of detection at a MS signal-to-noise ratio of 3 were 25, 54, and $34 \mathrm{nM}$ for glutathione, Cys and homocysteine, respectively, while limits of detection of $1.0,2.0$ and $1.3 \mu \mathrm{M}$ were obtained without any pretreatment on the same mass spectrometer.
Furthermore, the authors applied this method for the analyzes of glutathione in red blood cells and of Cys in plasma, which showed great potential for diagnosis.

By modifying AuNPs with special ligands that are enzymatic substrates, enzyme assay investigation by MS was realized. Kim developed a label-free assay of protein kinase on peptideconjugated AuNPs by using secondary-ion mass spectrometer, where the AuNPs acted as both signal enhancers and target concentrators [85]. Two Cys-terminated peptides with different lengths were conjugated to AuNPs based on the affinity between Au and thiol groups. The kinase reaction resulted in a 
straightforward change in the mass of the peptide substrates, where a mass shift equivalent to that of $\mathrm{HPO}_{3}(80 \mathrm{Da})$ could be observed. The phosphorylation efficiency was determined by the intensity ratio of the original unphosphorylated peptide peak and the phosphorylated peptide peak. This strategy holds advantages of label-free and reliable quantification results. Similarly, carbohydrate was modified with thiol and immobilized on the AuNPs for monitoring glycosyltransferase reactions using MS [86].

Modified AuNPs have also been used to amplify signals associated with DNA hybridization, allowing for sensitive detection of DNA, as shown in Figure 3 [87]. The AuNPs were modified with probe-DNA strand and small alkanethiol molecules. Target-DNA strands were immobilized on a glass or silicon wafer substrate through the capture strands. By incubating this substrate with the modified AuNPs, the probe strand could hybridize with the target strand. When these captured AuNPs were analyzed by NALDI-MS, alkanethiol molecules, which existed in large excess over the probe-DNA strands on the gold particle, were ionized and detected rather than the DNA strands. In this way, the DNA hybridization can be transduced to a highly amplified mass signal by observing a large number of small molecules instead of target DNA strands. As a result, detection limits as low as $100 \mathrm{pM}$ were achieved. By using distinct alkanethiol molecules to modify the AuNPs that carried different probe strands, this DNA hybridization amplification method could also be applied to the analysis of several target DNA strands simultaneously. A similar strategy was also applied for the MS signal amplification of target proteins by changing the probe strands on AuNPs to ligands or antibody molecules and the target strands to proteins [88].

AuNPs are also promising candidates as drug delivery agents into cells. Rotello et al. demonstrated that MS can be used for the analysis of cellular uptake based on the functionalized AuNPs with cationic or neutral surface ligands (Figure 4) [89]. The surface ligands could be viewed as 'mass barcodes' that allow AuNPs with different modifications to be simultaneously identified and quantified at levels as low as $30 \mathrm{pmol}$. Specifically, AuNPs were firstly incubated with cells for $6 \mathrm{~h}$ for cellular uptake. Afterwards, the phosphate-buffered saline solution was used to wash the cells to remove extra AuNPs that were not taken up by the cells. Transmission electron microscopy was used to verify the cellular uptake of AuNPs. The cells were then lysed and the AuNPs taken up by the cells were collected as part of the precipitate after centrifugation of the lysate. By analyzing the collected AuNPs with NALDI-MS, the 'mass barcodes' ligands were ionized and detected to demonstrate and quantify the AuNPs. This study has shown that the cellular uptake of the functionalized AuNPs is dependent on the functionality of the nanoparticle surfaces, suggesting that

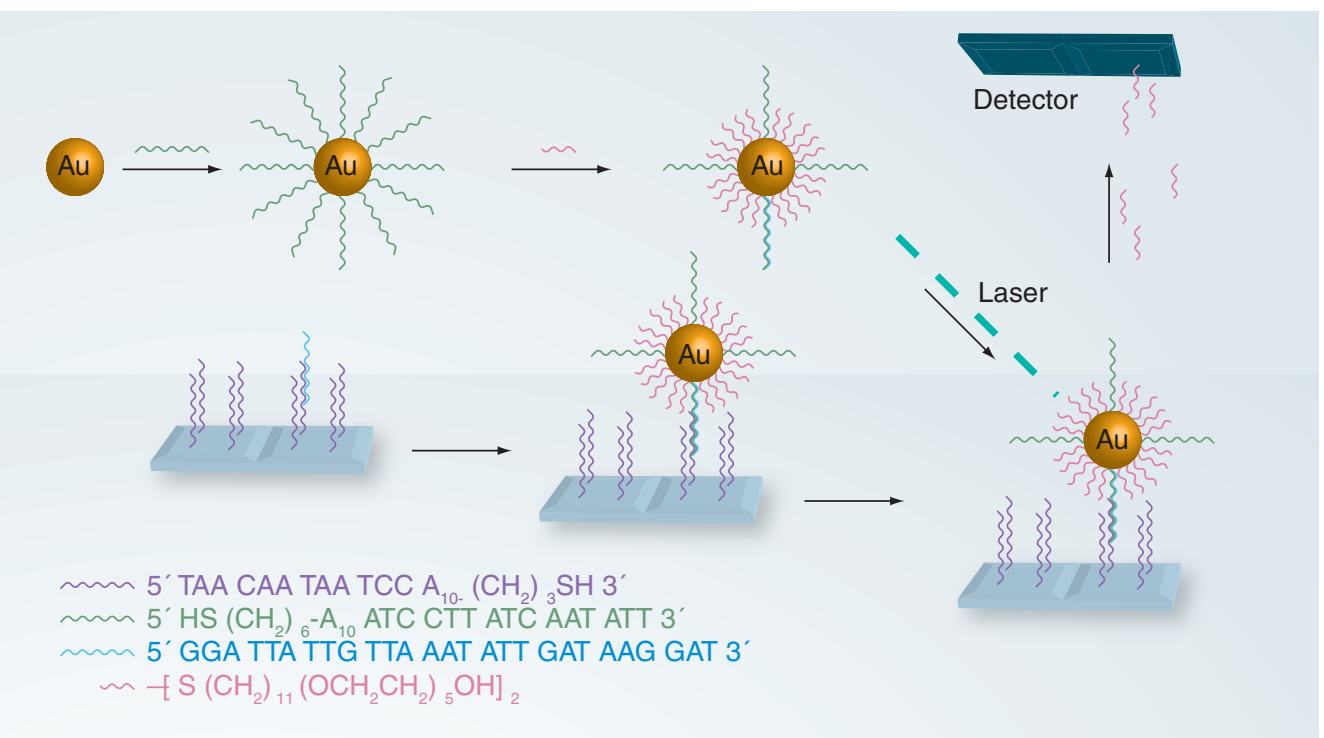

Figure 3. Functionalized DNA hybridization assay with nanomaterial-assisted laser desorption ionization-time of flight mass spectrometry.

Au: Gold.

Reproduced with permission from [87]. 


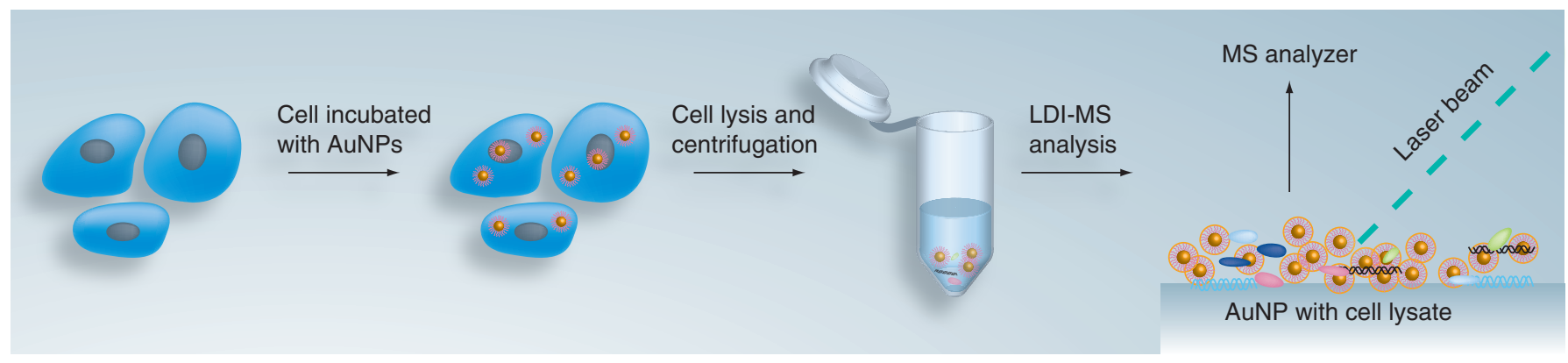

Figure 4. Gold nanoparticle cellular uptake and the analysis of the gold nanoparticles in cell lysates by nanomaterialassisted laser desorption ionization mass spectrometry.

AuNP: Gold nanoparticle; LDI: Laser desorption/ionization; MS: Mass spectrometry.

Reproduced with permission from [89].

differential cellular uptake and specific cell targeting might be possible if the appropriate surface functionalities are chosen.

\section{Nanomaterials to induce in-source reaction during the MALDI \& NALDI processes}

Oxidation of biomolecules plays an important role in many human diseases. The most important oxidative species in a human body are reactive oxygen species (ROS) acting both as deleterious and beneficial species [90]. Common ROS include superoxide anion $\left(\mathrm{O}_{2}^{--}\right)$, hydroxyl radical $\left(\bullet^{\circ} \mathrm{OH}\right)$ and peroxyl radicals $\left(\mathrm{ROO}^{\bullet}\right)$ [90]. At low/moderate concentrations, ROS can be important messengers for signal transduction, while at high concentration, ROS can induce oxidative damage to DNA, lipids and proteins, and these processes are usually described as oxidative stress [90]. These oxidation reactions take place not only during diseases such as cancer, cardiovascular troubles, diabetes and neurological disorders, but also naturally during aging [90].

Nanosemiconductor materials or QDs, characterized by separated valence and conduction bands with a moderate band gap, have been widely used to investigate the oxidation of molecules. Under light irradiation, electrons are excited from the valence band to the conduction band, generating oxidative holes and reductive electrons that can induce redox reaction with target molecules. Combining this photoreaction with the LDI process, on-line redox reactions on biomolecules were achieved during the MALDI-MS or NALDI-MS analysis. In particular, nano- $\mathrm{TiO}_{2}, \mathrm{Ga}_{2} \mathrm{O}_{3}$ or silicon was used as the substrate to deposit analytes and assist LDI. Upon laser irradiation, photo-induced redox reactions took place in the ionization source. Afterwards, the reactants as well as the products could be extracted by the electric field and analyzed in the mass spectrometer. Primary studies on in-source redox reactions during NALDI include oxidation of ferrocene derivatives and reduction of organic dyes during DIOS [91-93]. Recently, we employed $\mathrm{TiO}_{2}$ nanoparticle modified substrates to perform on-line redox reactions on biomolecules coupled with MS analysis [30-32]. The applications of this strategy involve on-line tagging of Cys residues by quinone compounds, in-source reduction of disulfide bonds in proteins and oxidation of peptides for $a, x$-fragmentation, as shown in Figure 5 [30-32].

Commercial $\mathrm{TiO}_{2}$ nanoparticles (Degussa $\mathrm{P} 25)$ were separated in ethanol $/ \mathrm{H}_{2} \mathrm{O}$ and deposited on a stainless steel target plate or on an aluminium foil. After drying in ambient conditions, the modified substrate was heated at $400^{\circ} \mathrm{C}$ for

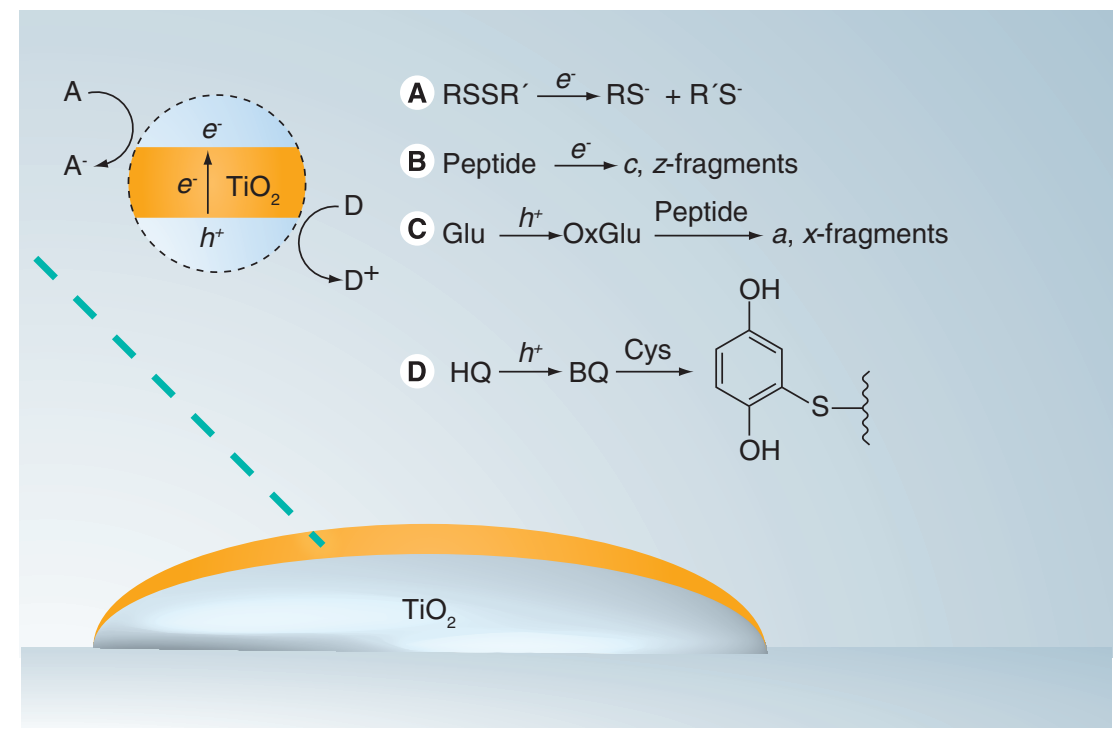

Figure 5. In-source redox reactions during the matrix-assisted laser desorption ionization or nanomaterial-assisted laser desorption ionization process [98,99]. (A) Reduction of disulfide bond; (B) peptide reductive C, $z$-fragmentation; (C) peptide oxidative $a, x$-fragmentation; and (D) Cys on-line tagging.

BQ: Benzoquinone; Cys: Cysteine; Glu: Glucose; $h^{+}$: Holes generated in the valence band of semiconductor under light irradiation; HQ: Hydroquinone; OxGlu: Oxidized glucose. 
$2 \mathrm{~h}$ to sinter the $\mathrm{TiO}_{2}$ nanoparticles and to form a stable photosensitive layer. For tagging Cys residues, Cys-containing peptides were deposited together with hydroquinone on the $\mathrm{TiO}_{2}$ spot [32] . Under UV irradiation, photo-generated holes oxidized hydroquinone to produce benzoquinone, which could further react with the thiol group on Cys-containing peptides to tag them concomitantly with the sample ionization, as shown in Figure 5. As a result, the peaks for both untagged and tagged peptides were observed together on one mass spectrum. This on-line tagging method was proven to be sensitive and highly specific for the synthetic peptides, protein digests and even protein mixture digests. For example, for peptides containing three Cys residues, signals coming from untagged, single, double and triple tagged peptides could all be observed in one spectrum, therefore providing a convenient method for counting free Cys residues present in peptides. The number of Cys residues in a peptide is important supplementary information in the process of database interrogation as it is needed for MS-based protein identification, to distinguish isobaric peptides. For example, $\beta$-lactoglobulin A was identified with a database searching score of 196 by considering the information on Cys content based on peptide mass fingerprinting method by using Mascot as the searching engine and SWISS-PROT as the database, whereas a score of only 151 was obtained without this information. Higher score value indicates a more accurate identification. Similarly, the database searching score was enhanced from 261 to 424 for bovine serum albumin.

For the reduction of disulfide bonds in proteins, glucose was deposited on the $\mathrm{TiO}_{2}$ layer to enhance the reductive ability of the semiconductor under light irradiation [30]. The hydroxyl group on glucose can extract the holes generated in the $\mathrm{TiO}_{2}$ substrate and free electrons. These electrons could then react with the disulfide bond and break this bond, as shown in Figure 5. When using human insulin (a protein containing three disulfide bonds), only the peak corresponding to the intact protein was observed on the classical MALDI-MS spectrum, while the fragments of A- and B-chains, disulfide bond cleavage products, were observed by using the in-source reduction strategy.

During the investigation of photoinduced reductions, it was found that $\mathrm{TiO}_{2}$ could also induce peptide regular fragmentation with the presence of glucose [31]. Specifically, glucose was oxidized by the holes, and the oxidized glucose could further oxidize the peptides and cleave the $\mathrm{C}_{a}-\mathrm{C}$ bond to generate $a, x$-fragments, as indicated in Figure 5. On the other hand, the electrons in the conduction band could directly react with peptides to induce the $c, z$-fragmentation on N-C bond (Figure 5). However, because there was no effective electron conductor in the reaction system, reduction could only happen on the surface of $\mathrm{TiO}_{2}$, while oxidation could also occur in the ionization plume due to the presence of glucose. As a result, stronger $a$-fragments were observed compared with the $c$-fragments. The $a, x$-fragmentation is usually relatively seldom observed when using common peptide dissociation strategies. The present $\mathrm{TiO}_{2}$-induced in-source peptide fragmentation can be useful for peptide sequencing.

\section{Conclusion \& future perspective}

This article shows how nanomaterials have been used for the ionization of molecules for MS detection, focusing on the applications of direct analysis of drug molecules, tissue imaging, enzyme assay and binding assays. This article also focuses on the major nanomaterials commonly used, namely silicon, gold and $\mathrm{TiO}_{2}$. Silicon is the most widely used inorganic materials to assist LDI, where high sensitivity has been achieved especially for small biomolecules. AuNPs are specifically used as both probes to selectively extract molecules of interest and as matrices to assist LDI. By modifying these nanoparticles with specific ligands or functional groups, selective characterization of low abundant biomolecules, and sensitive detection of proteins, DNA and cellular uptake can be realized.

The limitation of all matrix-free LDI and NALDI techniques is that the ionization efficiency decreases for large molecules. As discussed above, matrix-free LDI and NALDI are normally used for the investigation of small molecules (MW: $<2 \mathrm{kDa}$ ) of metabolites and medicine. In some research, the NALDI technology was used to ionize small proteins (MW: $\sim 20 \mathrm{kDa}$ ) [28]. However, it is not interesting to develop NALDI-related methods just for the ionization of biomacromolecules that can be adequately detected with the typical MALDI-MS strategy. In our view, it is worthy to further develop LDI in-source reactions for protein/ peptide tagging, oxidation and fragmentation by using $\mathrm{TiO}_{2}$ or QDs. However, in order to enhance the oxidation or fragmentations efficiency of protein/peptide, different additive molecules containing special functions, new hole scavenger candidates and matrix composition should be characterized. 
Semiconductor materials can also be coupled with electrospray ionization MS to achieve online oxidation of biomolecules, where ROS [94] of hydroxyl radical $\left({ }^{\circ} \mathrm{OH}\right)$ and peroxyl radicals $\left(\mathrm{ROO}^{\bullet}\right)$ can be generated, offering a novel strategy in the research of antioxidant filtration and disease mechanism study.

As another future perspective, novel functional mesoporous materials can be considered to facilitate biological reaction kinetics, owing to their major advantages of having large specific surface areas and uniform pore structure. Specifically, macroporous silica or titania can be used to immobilize enzymes for fast on-plate protein digestion [95-97], which can provide promising platforms for future tissue imaging of large biomolecules of interest.

Nanomaterial-assisted LDI has shown successful applications in metabolism investigation. The NALDI strategies were also combined with MS imaging techniques for the investigation of the distribution of medicine metabolites and proteins in tissues or cells. However, the study of NALD-MS imaging is still in the early stages. Further research in areas such as highly sensitive and selective imaging methods should be performed in the future.

\section{Financial \& competing interests disclosure}

The authors acknowledge funding provided by the Swiss National Science Foundation 200020-127142 and National Science Foundation of China 20925517. The authors have applied for patents in areas related to nanomaterial-assisted laser desorption ionization devices. The authors have no other relevant affliations or financial involvement with any organization or entity with a financial interest in or financial confict with the subject matter or materials discussed in the manuscript apart from those disclosed.

No writing assistance was utilized in the production of this manuscript.

\section{Executive summary}

- Matrix-assisted laser desorption ionization mass spectrometry is a very useful method for the analysis of large biomolecules including proteins, nucleic acids and peptides.

- Nanomaterial-assisted laser desorption ionization method has been performed by depositing analytes directly on the silicon-related substrate, gold nanoparticles, semiconducting particles and many other nanomaterials for the analysis of small molecules, such as therapeutic and diagnostic molecules.

- Compared with the typical matrix-assisted laser desorption ionization, nanomaterial-assisted laser desorption ionization demonstrates a very clean background in the low mass range and more homogeneous surface geometry, making it suitable for quantitative analysis.

- Functionalized nanoparticles can be used both as probes to extract target molecules of interest and as matrices to assist laser desorption ionization.

- Mass spectrometry imaging has been used as a label-free protocol to investigate the in situ distribution of peptides, proteins, metabolites and medicines in tissues and cells.

- Semiconducting nanomaterials act as photosensitive matrices to induce efficient in-source redox reactions, and can be further applied in protein oxidation for metabolomics and protein/peptide fragmentation and identification.

\section{Bibliography}

Papers of special note have been highlighted as: " of interest

= of considerable interest

1 Karas M, Bachmann D, Bahr U, Hillenkamp F: Matrix-assisted ultravioletlaser desorption of nonvolatile compounds. Int. J. Mass Spectrom. Ion Process. 78, 53-68 (1987).

2 Yamashita M, Fenn JB: Electrospray ion-source - another variation on the free-jet theme. J. Phys. Chem. 88(20), 4451-4459 (1984).

3 Karas M, Gluckmann M, Schafer J: Ionization in matrix-assisted laser desorption/ ionization: singly charged molecular ions are the lucky survivors. J. Mass Spectrom. 35(1), 1-12 (2000).

4 Tanaka K, Waki H, Ido Y et al.: Protein and polymer analyses up to m/z 100000 by laser ionization time-of-flight mass spectrometry. Rapid Commun. Mass Spectrom. 2(8), 151-153 (1988). "n The first laser desorption/ionization (LDI) publication.

5 Wei J, Buriak JM, Siuzdak G: Desorptionionization mass spectrometry on porous silicon. Nature 399(6733), 243-246 (1999).

- The most widely used LDI method.

6 Huikko K, Ostman P, Sauber C et al.: Feasibility of atmospheric pressure desorption/ ionization on silicon mass spectrometry in analysis of drugs. Rapid Commun. Mass Spectrom. 17(12), 1339-1343 (2003).

7 Laiko VV, Taranenko NI, Berkout VD, Musselman BD, Doroshenko VM: Atmospheric pressure laser desorption/ ionization on porous silicon. Rapid Commun. Mass Spectrom. 16(18), 1737-1742 (2002).

8 Ostman P, Pakarinen JMH, Vainiotalo P, Franssila S, Kostiainen R, Kotiaho T: Minimum proton affinity for efficient ionization with atmospheric pressure desorption/ionization on silicon mass spectrometry. Rapid Commun. Mass Spectrom. 20(24), 3669-3673 (2006).
9 Pihlainen K, Grigoras K, Franssila S, Ketola R, Kotiaho T, Kostiainen R: Analysis of amphetamines and fentanyls by atmospheric pressure desorption/ionization on silicon mass spectrometry and matrixassisted laser desorption/ionization mass spectrometry and its application to forensic analysis of drug seizures. J. Mass Spectrom. 40(4), 539-545 (2005).

10 Yoon SH, Gamage CM, Gillig KJ, Wysocki VH: Kinetics of surface-induced dissociation of $\mathrm{N}\left(\mathrm{CH}_{3}\right)_{4}{ }^{+}$and $\mathrm{N}\left(\mathrm{CD}_{3}\right)_{4}{ }^{+}$using silicon nanoparticle assisted laser desorption/ ionization and laser desorption/ionization. J. Am. Soc. Mass Spectrom. 20(6), 957-964 (2009).

11 Bhattacharya SH, Raiford TJ, Murray KK: Infrared laser desorption/ionization on silicon. Anal. Chem. 74(9), 2228-2231 (2002).

12 Go EP, Apon JV, Luo G et al:: Desorption/ ionization on silicon nanowires. Anal. Chem. 77(6), 1641-1646 (2005). 
13 Lee CS, Kang KK, Kim JH et al.: Analysis of small molecules by desorption/ionization on mesoporous silicate (DIOM)-mass spectrometry (MS). Microporous Mesoporous Mater. 98(1-3), 200-207 (2007).

14 Lee CS, Song HM, Kang KK, Choi CH, Rhee HK, Kim BG: Desorption/ionization on mesoporous silicate (DIOM)-mass spectrometry (MS) and ultrafiltration for target-based drug screening. Biochip J. 1(1), 43-48 (2007).

15 Gorecka-Drzazga A, Bargiel S, Walczak R et al.: Desorption/ionization mass spectrometry on porous silicon dioxide. Sens. Actuators B Chem. 103(1-2), 206-212 (2004).

16 Novikov A, Caroff M, Della-Negra S et al.: Matrix-implanted laser desorption/ionization mass spectrometry. Anal. Chem. 76(24), 7288-7293 (2004).

17 Wu HP, Yu CJ, Lin CY, Lin YH, Tseng WL: Gold nanoparticles as assisted matrices for the detection of biomolecules in a high-salt solution through laser desorption/ionization mass spectrometry. J. Am. Soc. Mass Spectrom. 20(5), 875-882 (2009).

18 Kawasaki H, Sugitani T, Watanabe T, Yonezawa T, Moriwaki H, Arakawa R: Layer-by-layer self-assembled mutilayer films of gold nanoparticles for surface-assisted laser desorption/ionization mass spectrometry. Anal. Chem. 80 (19), 7524-7533 (2008).

19 Love JC, Estroff LA, Kriebel JK, Nuzzo RG, Whitesides GM: Self-assembled monolayers of thiolates on metals as a form of nanotechnology. Chem. Rev. 105, 1103-1169 (2005).

20 Castellana ET, Russell DH: Tailoring nanoparticle surface chemistry to enhance laser desorption ionization of peptides and proteins. Nano Lett. 7, 3023-3025 (2007).

21 Duan JC, Linman MJ, Chen CY, Cheng QJ: CHCA-modified Au nanoparticles for laser desorption ionization mass spectrometric analysis of peptides. J. Am. Soc. Mass Spectrom. 20(8), 1530-1539 (2009).

22 Chen LC, Mori K, Hori H, Hiraoka K: $\mathrm{Au}$-assisted visible laser MALDI. Int. J. Mass Spectrom. 279(1), 41-46 (2009).

23 Chen LC, Ueda T, Sagisaka M, Hori H, Hiraoka K: Visible laser desorption/ionization mass spectrometry using gold nanorods. J. Phys.Chem. C 111(6), 2409-2415 (2007).

24 Chen LC, Yonehama J, Ueda T, Hori H, Hiraoka K: Visible-laser desorption/ ionization on gold nanostructures. J. Mass Spectrom. 42(3), 346-353 (2007).

25 Shan Z, Han L, Yuan MJ et al.: Mesoporous tungsten titanate as matrix for matrix-assisted laser desorption/ionization time-of-flight mass spectrometry analysis of biomolecules. Anal. Chim. Acta 593(1), 13-19 (2007).
26 Castro AL, Madeira PJA, Nunes MR, Costa FM, Florencio MH: Titanium dioxide anatase as matrix for matrix-assisted laser desorption/ ionization analysis of small molecules. Rapid Commun. Mass Spectrom. 22(23), 3761-3766 (2008).

27 Chen CT, Chen YC: Molecularly imprinted $\mathrm{TiO}_{2}$-matrix-assisted laser desorption/ ionization mass spectrometry for selectively detecting $\alpha$-cyclodextrin. Anal. Chem. 76(5), 1453-1457 (2004).

28 Chen CT, Chen YC: Desorption/ionization mass spectrometry on nanocrystalline titania sol-gel-deposited films. Rapid Commun. Mass Spectrom. 18 (17), 1956-1964 (2004).

29 Lachheb H, Puzenat E, Houas A et al.: Photocatalytic degradation of various types of dyes (alizarin S, crocein orange G, methyl red, congo red, methylene blue) in water by UV-irradiated titania. Appl. Catal. B-Environ. 39(1), 75-90 (2002).

30 Qiao L, Bi HY, Busnel JM, Liu BH, Girault $\mathrm{HH}$ : In-source photocatalytic reduction of disulfide bonds during laser desorption ionization. Chem. Commun.(47), 6357-6359 (2008).

31 Qiao L, Bi HY, Busnel JM et al.: Photocatalytic redox reactions for in-source peptide fragmentation. Chem. Eur. J. 15(27), 6711-6717 (2009).

- Peptide $a, x$-fragmentation during the LDI process, valuable for peptide sequencing.

32 Qiao L, Roussel C, Wan JJ et al.: MALDI in-source photooxidation reactions for online peptide tagging. Angew. Chem. Int. Ed. Engl. 47(14), 2646-2648 (2008).

- Matrix-assisted LDI (MALDI)-based on-line cysteine tagging strategy, valuable for protein accurate identification.

33 Sunner J, Dratz E, Chen YC: Graphite surface assisted laser desorption/ionization time-offlight mass-spectrometry of peptides and proteins from liquid solutions. Anal. Chem. 67(23), 4335-4342 (1995).

34 Wen HS, Tsai LF, Liu CW et al.: Investigation of effects attributed to spherical carbon nanomaterials in proteomic matrix-assisted laser desorption ionizationtime of flight mass spectrometer. 1-3, 1019-1021 (2007). Presented at: 7th IEEE Conference on Nanotechnology. Hong Kong, China, 2-5 August 2007.

35 Xu SY, Li YF, Zou HF, Qiu JS, Guo Z, Guo BC: Carbon nanotubes as assisted matrix for laser desorption/ionization time-of-flight mass spectrometry. Anal. Chem. 75, 6191-6195 (2003).

36 Zhang J, Wang HY, Guo YL: Amino acids analysis by MALDI mass spectrometry using carbon nanotube as matrix. Chinese J. Chem. 23(2), 185-189 (2005).

37 Najam-ul-Haq M, Rainer M, Huck CW, Hausberger P, Kraushaar H, Bonn GK: Nanostructured diamond-like carbon on digital versatile disc as a matrix-free target for laser desorption/ionization mass spectrometry. Anal. Chem. 80(19), 7467-7472 (2008).

38 Shenar N, Martinez J, Enjalbal C: Laser desorption/ionization mass spectrometry on porous silica and alumina for peptide mass fingerprinting. J. Am. Soc. Mass Spectrom. 19(5), 632-644 (2008).

39 Watanabe T, Kawasaki H, Yonezawa T, Arakawa R: Surface-assisted laser desorption/ ionization mass spectrometry (SALDI-MS) of low molecular weight organic compounds and synthetic polymers using zinc oxide $(\mathrm{ZnO})$ nanoparticles. J. Mass Spectrom. 43(8), 1063-1071 (2008).

40 Shin WJ, Shin JH, Song JY, Han SY: Effects of $\mathrm{ZnO}$ nanowire length on surface-assisted laser desorption/ionization of small molecules. J. Am. Soc. Mass Spectrom. 21(6), 989-992 (2010)

41 Chen ZM, Geng ZR, Shao DL, Mei YH, Wang ZL: Single-crystalline EUF3 hollow hexagonal microdisks: synthesis and application as a background-free matrix for MALDI-TOF-MS analysis of small molecules and polyethylene glycols. Anal. Chem. 81(18), 7625-7631 (2009).

42 Hua L, Chen JR, Ge L, Tan SN: Silver nanoparticles as matrix for laser desorption/ ionization mass spectrometry of peptides. J. Nano. Res. 9, 1133-1138 (2007).

43 Chiang CK, Yang ZS, Lin YW, Chen WT, Lin HJ, Chang HT: Detection of proteins and protein-ligand complexes using HGTE nanostructure matrixes in surface-assisted laser desorption/ionization mass spectrometry. Anal. Chem. 82(11), 4543-4550 (2010).

44 Kawasaki H, Yonezawa T, Watanabe T, Arakawa R: Platinum nanoflowers for surfaceassisted laser desorption/ionization mass spectrometry of biomolecules. J. Phys. Chem. C 111, 16278-16283 (2007).

45 Taira S, Kitajima K, Katayanagi H, Ichiishi E, Ichiyanagi Y: Manganese oxide nanoparticleassisted laser desorption/ionization mass spectrometry for medical applications. Sci. Technol. Adv. Mater. 10(3), 034602 (2009).

46 Chen WY, Chen YC: Affinity-based mass spectrometry using magnetic iron oxide particles as the matrix and concentrating probes for SALDI MS analysis of peptides and proteins. Anal. BioAnal. Chem. 386(3), 699-704 (2006). 
47 Szabo Z, Vallant RM, Takatsy A et al:: Laser desorption/ionization mass spectrometric analysis of small molecules using fullerenederivatized silica as energy-absorbing material. J. Mass Spectrom. 45(5), 545-552 (2010).

48 Seino T, Sato H, Yamamoto A, Nemoto A, Torimura M, Tao H: Matrix-free laser desorption/ionization-mass spectrometry using self-assembled germanium nanodots. Anal. Chem. 79(13), 4827-4832 (2007).

49 Bi HY, Girault HH: Mass spectrometry based analysis and bioanalysis. Thèse Ecole Polytechnique Fédérale de Lausanne (EPFL). Lausanne, Switzerland, No. 4741 (2010).

50 Chiang CK, Chiang NC, Lin ZH, Lan GY, Lin YW, Chang HT: Nanomaterial-based surface-assisted laser desorption/ionization mass spectrometry of peptides and proteins. J. Am. Soc. Mass Spectrom. 21(7), 1204-1207 (2010).

51 Shenar N, Cantel S, Martinez J, Enjalbal C: Comparison of inert supports in laser desorption/ionization mass spectrometry of peptides: pencil lead, porous silica gel, DIOS-chip and NALDI target. Rapid Commun. Mass Spectrom. 23(15), 2371-2379 (2009).

52 Tang HW, Ng KM, Lu W, Che CM: Ion desorption efficiency and internal energy transfer in carbon-based surface-assisted laser desorption/ionization mass spectrometry: desorption mechanism(s) and the design of SALDI substrates. Anal. Chem. 81(12), 4720-4729 (2009).

53 Guo Z, Ganawi AAA, Liu Q, He L: Nanomaterials in mass spectrometry ionization and prospects for biological application. Anal. Bioanal. Chem. 384(3), 584-592 (2006).

54 Chiu TC, Huang LS, Lin PC et al:: Nanomaterial based affinity matrix-assisted laser desorption/ionization mass spectrometry for biomolecules and pathogenic bacteria. Recent Pat. Nanotechnol. 1(2), 99-111 (2007).

55 Vidova V, Novak P, Strohalm M, Pol J, Havlicek V, Volny M: Laser desorptionionization of lipid transfers: tissue mass spectrometry imaging without MALDI matrix. Anal. Chem. 82(12), 4994-4997 (2010).

- A very interesting LDI-mass spectrometry (MS) imaging strategy, where the nanomaterial-assisted LDI (NALDI) plate was firstly used as a substrate for the imprinting of tissue section for MS imaging.

56 Kraj A, Dylag T, Gorecka-Drzazga A, Bargiel S, Dziuban J, Silberring J: Desorption/ionization on silicon for small molecules: a promising alternative to MALDI TOF. Acta Biochimica Polonica 50 (3), 783-787 (2003).
57 Budimir N, Blais JC, Fournier F, Tabet JC: Desorption/ionization on porous silicon mass spectrometry (DIOS) of model cationized fatty acids. J. Mass Spectrom. 42(1), 42-48 (2007).

58 Budimir N, Blais JC, Fournier F, Tabet JC: The use of desorption/ionization on porous silicon mass spectrometry for the detection of negative ions for fatty acids. Rapid Commun. Mass Spectrom. 20(4), 680-684 (2006).

59 Wen XJ, Dagan S, Wysocki VH: Smallmolecule analysis with silicon-nanoparticleassisted laser desorption/ionization mass spectrometry. Anal. Chem. 79(2), 434-444 (2007).

60 Kraj A, Jarzebinska J, Gorecka-Drzazga A, Dziuban J, Silberring J: Identification of catecholamines in the immune system by desorption/ionization on silicon. Rapid Commun. Mass Spectrom.20(13), 1969-1972 (2006).

61 Arakawa R, Shimomae Y, Morikawa $\mathrm{H}$, Ohara K, Okuno S: Mass spectrometric analysis of low molecular mass polyesters by laser desorption/ionization on porous silicon. J. Mass Spectrom.39(8), 961-965 (2004).

62 Okunoa S, Wada Y, Arakawa R: Quantitative analysis of polypropyleneglycol mixtures by desorption/ionization on porous silicon mass spectrometry. Int. J. Mass Spectrom. 241(1), 43-48 (2005).

63 Go EP, Shen ZX, Harris K, Siuzdak G: Quantitative analysis with desorption/ ionization on silicon mass spectrometry using electrospray deposition. Anal. Chem. 75(20), 5475-5479 (2003).

64 Wall DB, Finch JW, Cohen SA: Quantification of codeine by desorption/ ionization on silicon time-of-flight mass spectrometry and comparisons with liquid chromatography/mass spectrometry. Rapid Commun. Mass Spectrom.18(12), 1403-1406 (2004).

65 Pan CS, Xu SY, Hu LG et al:: Using oxidized carbon nanotubes as matrix for analysis of small molecules by MALDI-TOF MS. J. Am. Soc. Mass Spectrom. 16(6), 883-892 (2005).

66 Lo CY, Lin JY, Chen WY, Chen CT, Chen YC: Surface-assisted laser desorption/ ionization mass spectrometry on titania nanotube arrays. J. Am. Soc. Mass Spectrom. 19(7), 1014-1020 (2008).

67 Su CL, Tseng WL: Gold nanoparticles as assisted matrix for determining neutral small carbohydrates through laser desorption/ ionization time-of-flight mass spectrometry. Anal. Chem. 79(4), 1626-1633 (2007).

68 Shen ZX, Go EP, Gamez A et al: A mass spectrometry plate reader: monitoring enzyme activity and inhibition with a desorption/ionization on silicon (DIOS) platform. Chembiochem. 5(7), 921-927 (2004).

69 Thomas JJ, Shen ZX, Crowell JE, Finn MG, Siuzdak G: Desorption/ionization on silicon (DIOS): a diverse mass spectrometry platform for protein characterization. Proc. Natl Acad. Sci. USA 98(9), 4932-4937 (2001).

"- LDI-based enzyme activity investigation with high throughput and efficiency.

70 Li Q, Ricardo A, Benner SA, Winefordner JD, Powell DH: Desorption/ ionization on porous silicon mass spectrometry studies on pentose-borate complexes. Anal. Chem. 77(14), 4503-4508 (2005).

"- DIOS-MS as an ideal platform for the investigation of reaction equilibrium.

71 van Hove ERA, Smith DF, Heeren RMA: A concise review of mass spectrometry imaging. J. Chromatogr. A 1217(25), 3946-3954 (2010).

72 Chughtai K, Heeren RMA: Mass spectrometric imaging for biomedical tissue analysis. Chem. Rev. 110(5), 3237-3277 (2010).

73 McDonnell LA, Heeren RMA: Imaging mass spectrometry. Mass Spectrom. Rev. 26(4), 606-643 (2007).

74 Liu Q, Guo Z, He L: Mass spectrometry imaging of small molecules using desorption/ ionization on silicon. Anal. Chem. 79(10), 3535-3541 (2007).

75 Ageta H, Asai S, Sugiura Y, Goto-Inoue N, Zaima N, Setou M: Layer-specific sulfatide localization in rat hippocampus middle molecular layer is revealed by nanoparticleassisted laser desorption/ionization imaging mass spectrometry. Med. Mol. Morphol. 42(1), 16-23 (2009).

76 Taira S, Sugiura Y, Moritake S, Shimma S, Ichiyanagi Y, Setou M: Nanoparticle-assisted laser desorption/ionization based mass imaging with cellular resolution. Anal. Chem. 80(12), 4761-4766 (2008).

Wan JJ, Qian K, Qiao L et al.: $\mathrm{TiO}_{2}$-modified macroporous silica foams for advanced enrichment of multi-phosphorylated peptides. Chem. Eur. J. 15(11), 2504-2508 (2009).

78 Blacken GR, Volny M, Vaisar T, Sadilek M, Turecek F: In situ enrichment of phosphopeptides on MALDI plates functionalized by reactive landing of zirconium(IV)- $N$-propoxide ions. Anal. Chem. 79(14), 5449-5456 (2007).

79 Bi HY, Qiao L, Busnel JM, Devaud V, Liu $\mathrm{BH}$, Girault $\mathrm{HH}: \mathrm{TiO}_{2}$ printed aluminum foil: single-use film for a laser desorption/ ionization target plate. Anal. Chem. 81(3), 1177-1183 (2009). 
80 Qiao L, Roussel C, Wan JJ, Yang PY, Girault HH, Liu BH: Specific on-plate enrichment of phosphorylated peptides for direct MALDI-TOF MS analysis. J. Proteome Res. 6(12), 4763-4769 (2007).

81 Qiao LA, Bi HY, Busnel JM et al.: Controlling the specific enrichment of multi-phosphorylated peptides on oxide materials: aluminium foil as a target plate for laser desorption ionization mass spectrometry. Chem. Sci. 1(3), 374-382 (2010).

82 Lee KH, Chiang CK, Lin ZH, Chang HT: Determining enediol compounds in tea using surface-assisted laser desorption/ionization mass spectrometry with titanium dioxide nanoparticle matrices. Rapid Commun. Mass Spectrom. 21(13), 2023-2030 (2007).

83 Huang YF, Chang HT: Nile red-adsorbed gold nanoparticle matrixes for determining aminothiols through surface-assisted laser desorption/ionization mass spectrometry. Anal. Chem. 78(5), 1485-1493 (2006).

84 Huang YF, Chang HT: Analysis of adenosine triphosphate and glutathione through gold nanoparticles assisted laser desorption/ ionization mass spectrometry. Anal. Chem. 79(13), 4852-4859 (2007).

85 Kim YP, Oh E, Oh YH, Moon DW, Lee TG, Kim HS: Protein kinase assay on peptideconjugated gold nanoparticles by using secondary-ion mass spectrometric imaging. Angew. Chem. Int. Ed. Engl. 46, 6816-6819 (2007).

86 Nagahori N, Nishimura SI: Direct and efficient monitoring of glycosyltransferase reactions on gold colloidal nanoparticles by using mass spectrometry. Chem. Eur. J. 12(25), 6478-6485 (2006).
87 Qiu F, Jiang DW, Ding YB, Zhu J,

Huang LL: Monolayer-barcoded nanoparticles for on-chip DNA hybridization assay. Angew. Chem. Int. Ed. Engl. 47(27), 5009-5012 (2008).

- MS-based signal amplification method for DNA hybridization investigation.

88 Lee JR, Lee A, Kim SK, Kim KP, Park HS, Yeo WS: Mass spectrometry signal amplification method for attomolar detection of antigens using small-molecule-tagged gold microparticles. Angew. Chem. Int. Ed. Engl. 47(49), 9518-9521 (2008).

89 Zhu ZJ, Ghosh PS, Miranda OR, Vachet RW, Rotello VM: Multiplexed screening of cellular uptake of gold nanoparticles using laser desorption/ionization mass spectrometry. J. Am.Chem. Soc. 130(43), 14139-14143 (2008).

- Analysis of cellular uptake of nanoparticles based on MS.

90 Valko M, Leibfritz D, Moncol J, Cronin MTD, Mazur M, Telser J: Free radicals and antioxidants in normal physiological functions and human disease. Int. J. Biochem. Cell Biol. 39(1), 44-84 (2007).

91 Okuno S, Arakawa R, Wada Y: Reduction of $\mathrm{Cu}(\mathrm{II})$ and riboflavin in DIOS mass spectrometry. J. Mass Spectrom. Soc. Jpn. 52(1), 13-20 (2004).

92 Okuno S, Nakano M, Matsubayashi G, Arakawa R, Wada Y: Reduction of organic dyes in matrix-assisted laser desorption/ ionization and desorption/ionization on porous silicon. Rapid Commun. Mass Spectrom. 18(23), 2811-2817 (2004).
93 Okuno S, Oka K, Arakawa R: Oxidation of ferrocene derivatives in desorption/ionization on porous silicon. Anal. Sci. 21(12), 1449-1451 (2005).

94 Finkel T, Holbrook NJ: Oxidants, oxidative stress and the biology of ageing. Nature 408(6809), 239-247 (2000).

95 Bi HY, Qiao L, Busnel JM, Liu BH, Girault $\mathrm{HH}$ : Kinetics of proteolytic reactions in nanoporous materials. J. Proteome Res. 8(10), 4685-4692 (2009).

96 Qiao L, Liu Y, Hudson SP, Yang PY, Magner E, Liu BH: A nanoporous reactor for efficient proteolysis. Chem. Eur. J. 14(1), 151-157 (2008).

97 Qian K, Wan JJ, Qiao L et al.: Macroporous materials as novel catalysts for efficient and controllable proteolysis. Anal. Chem. 81(14), 5749-5756 (2009).

98 Abonnenc M, Qiao LA, Liu BH, Girault HH: Electrochemical aspects of electrospray and laser desorption/ionization for mass spectrometry. Ann. Rev. Anal. Chem. 3, 231-254 (2010).

99 Girault H, Liu BH, Qiao LA et al: Electrochemical reactions and ionization processes. Euro. J. Mass Spectrom. 16(3), 341-349 (2010). 